\title{
Polar NURBS Surface with Curvature Continuity
}

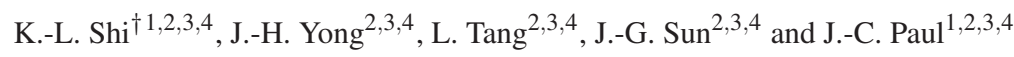 \\ ${ }^{1}$ Inria, Villers-lès-Nancy, France \\ ${ }^{2}$ School of Software, Tsinghua University, Beijing, China \\ ${ }^{3}$ Key Laboratory for Information System Security, Ministry of Education of China, Beijing, China \\ ${ }^{4}$ Tsinghua National Laboratory for Information Science and Technology, Beijing, China
}

\begin{abstract}
Polar NURBS surface is a kind of periodic NURBS surface, one boundary of which shrinks to a degenerate polar point. The specific topology of its control-point mesh offers the ability to represent a cap-like surface, which is common in geometric modeling. However, there is a critical and challenging problem that hinders its application: curvature continuity at the extraordinary singular pole. We first propose a sufficient and necessary condition of curvature continuity at the pole. Then, we present constructive methods for the two key problems respectively: how to construct a polar NURBS surface with curvature continuity and how to reform an ordinary polar NURBS surface to curvature continuous. The algorithms only depend on the symbolic representation and operations of NURBS, and they introduce no restrictions on the degree or the knot vectors. Examples and comparisons demonstrate the applications of the curvature-continuous polar NURBS surface in hole-filling and free-shape modeling.

Categories and Subject Descriptors (according to ACM CCS): I.3.5 [Computer Graphics]: Computational Geometry and Object Modeling—Curve, surface, solid, and object representations; Splines
\end{abstract}

\section{Introduction}

Most CAD systems and many geometric modeling systems in computer graphics represent free-form surfaces by NURBS (non-uniform rational B-splines) and its special case NUPBS (non-uniform polynomial B-splines). They have become de facto industrial standards for model representation, processing and data exchange [Far97, PT97a]. However, the rectangular parametric domain also has been pointed out not to be well-suited for capping disc-shaped circular regions [MP11, SYSP11], which are common in industrial geometric modeling, for example, the head of an airplane, the end of an aerofoil or a screw propeller (see Figure 1). A natural way to adapt the quadrilateral untrimmed NURBS surface to a cap-like shape is as follows. First, roll up the surface and glue two opposite boundary edges together to form a periodic NURBS surface. Then, shrink a loop edge to a single polar point as shown in Figure 2. The

$\dagger$ The research was partly supported by Chinese 973 Program (2010CB328001) and Chinese 863 Program (2012AA040902). The second author was supported by the NSFC $(61035002,61272235)$. The fifth author was supported by the NSFC $(61063029,61173077)$.
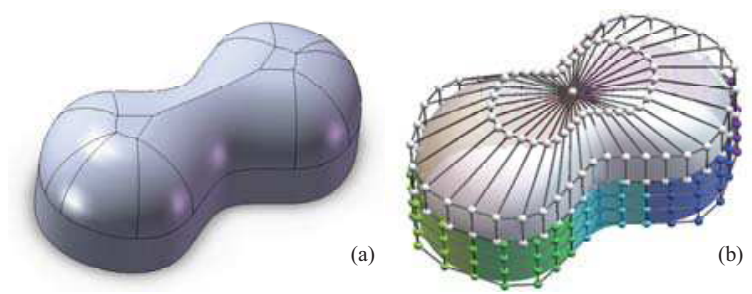

Figure 1: A transitional structure of a mechanical part designed by SolidWorks 2007 in (a). The 14 surface patches in (a) can be replaced by a single polar NURBS surface in (b).

result is called the polar NURBS surface. It makes up for the lack of ability of standard NURBS to represent a cap-like loop-bounded surface. Not only does this specific topology extend the application of NURBS, but also it preserves the NURBS form for CAD compatibility. It can be converted to a standard NURBS by knot-insertion. The conversion is bidirectional and theoretically free from error. It is also supported by the industrial standard IGES 5.0 [KR90]. 

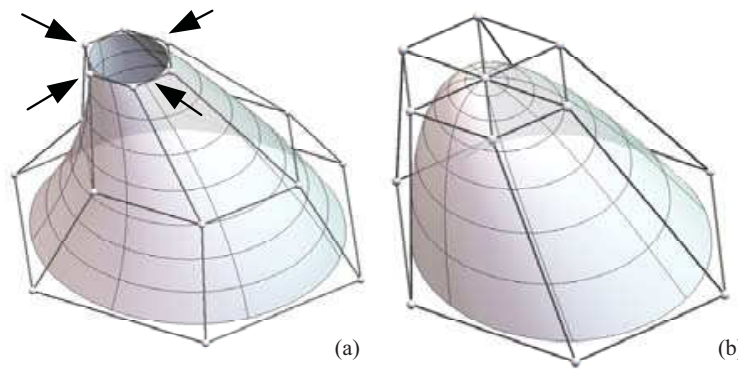

(b)

Figure 2: (a) A non-polar periodic NURBS surfaces; (b) A polar NURBS surface which has triangular fans in a polar configuration of its control points.

However, at the pole of the polar NURBS surface, there is still a challenging issue about curvature continuity $\left(G^{2}\right.$ continuity), which is essential and sometimes the minimum standard in geometric modeling such as class-A car-body design. At an interior point of a polar NURBS surface, the infimum order of parametric continuity, which is determined by the multiplicity of knots, has nothing different from ordinary periodic NURBS surface. However at the pole, which is actually a degenerate boundary edge, continuity also depends on the surrounding control points. The polar curvature of a non-well-formed polar NURBS surface is usually multivalued and that violates curvature continuity at the pole (see Figure 7). This problem prevents the polar NURBS surface from achieving higher-order continuity in practice. In order to solve the problem, we analyze the local differential feature of the singular pole and then propose methods to construct polar NURBS surfaces with curvature continuity. To be specific, the contributions of this paper are as follows. For the polar NURBS surface, we

- propose a sufficient and necessary condition of the polar curvature continuity;

- present a method to construct the control points that surround the pole to satisfy curvature continuity;

- present a method to reform a non-curvature-continuous polar NURBS surface to curvature-continuous, preserving the overall shape of the surface.

Not like other subdivision or spline-based methods, our work focuses on the relation between curvature continuity and radial cross-boundary derivatives in the NURBS form. All the procedures are based on the symbolic representation and operations of NURBS [PT97b], which introduce no restrictions on the input, for example, the degree and the knot vectors can be arbitrary.

Overview. After reviewing the related work in Section 2 and preliminaries in Section 3, we start from a sufficient and necessary condition of curvature continuity at the pole in Section 4. Based on the condition, Sections 5 and 6 then give a construction method and a reformation algorithm, respectively. The applications of $n$-sided hole filling and free-shape design in Section 7 illustrate the practicability of the curvature-continuous polar NURBS surfaces in CG and CAD. Section 8 concludes the paper.

\section{Related work}

Some methods of shrinking one edge of a NURBS surface to a single point have been proposed since the introduction of NURBS. For example, in Ref. [PT97a] §8.7, a method was presented to construct a three-sided spherical surface with one degenerate boundary. Ref. [SYSP10] introduced a polar parametric domain in blending multiple surfaces around an $n$-sided region. In Ref. [SYSP11], the periodic B-spline surface was used to fill $n$-sided holes. They assumed that the polar curvature is zero to avoid the problem of curvature continuity at the pole. Besides the NURBS form, studies on $G^{2}$ continuity at the extraordinary vertices have also yielded substantial results by subdivision [KMP06, KP07, Lev06, MP09]. However as discussed in Ref. [MP11], those subdivision methods do not readily fit into existing CAD frameworks, and the construction process, which may consist of an infinite sequence of surfaces, is unfriendly in further geometric processing such as intersection and trimming. There are also many methods to generate curvature-continuous splines from coarse quad meshes, for example, Hartmut and Prautzsch's free-form splines [Pra97], Karčiauskas and Peters' guided spline surfaces [KP09] and Reif's TURBS [Rei98]. Recently, Myles and Peters [MP11] gave a spline solution to the problem of curvature continuity for polar configurations in quad-dominant meshes. Their $C^{2}$ polar spline surface consists of one tensor-product spline of degree $(3,6)$, which is bi- $3 C^{2}$ compatible. It is more CADfriendly than polar subdivision. However, in real industrial CAD/CAM applications, we normally cannot limit the knot vectors, the degrees and the weights to this single pattern, and moreover, especially for mechanical modeling, the inputs are usually trimmed NURBS surface patches instead of two-manifold quad-dominant meshes. The degrees of the input patches may be different, and therefore the control points of two adjacent surfaces may not coincide even though they share the same edge geometrically. From a different aspect, this paper presents a more adaptive solution especially for $\mathrm{CG} / \mathrm{CAD} / \mathrm{CAM}$ modeling. The methods are derived from the symbolic representation of the cross-boundary derivative curves, which will not be limited by the inconsistent control meshes, degrees or knot vectors. In implementation, the symbolic operators can be applicable to both NURBS and NUPBS without changing the formulae.

\section{Definitions}

The periodic NURBS surface discussed in this paper is a NURBS surface that has a periodic knot sequence in only one parametric coordinate. It can be formulated as follows.

$$
\mathbf{S}^{w}(u, v)=\sum_{i, j} \tilde{N}_{i, p}(u) N_{j, q}(v) \mathbf{P}^{w}[i, j]
$$




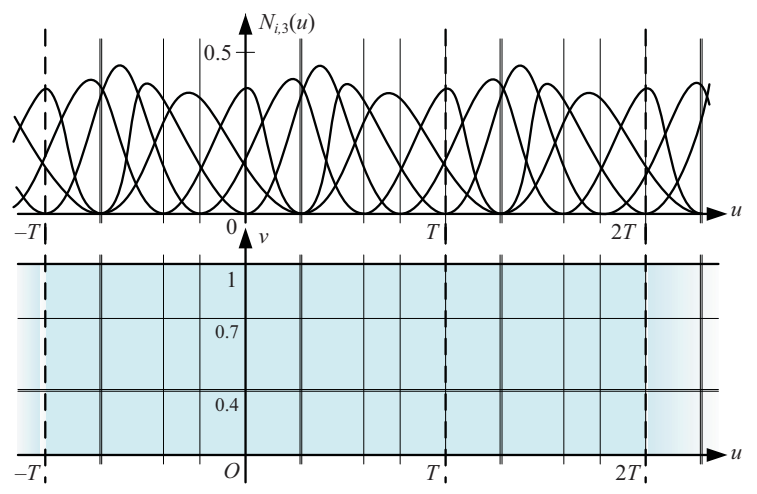

Figure 3: The periodic knot sequence in u-direction of the domain (blue band) and the corresponding basis functions.

Parameter $(u, v)$ is defined in a horizontal band in Euclidean space (see Figure 3). $\mathbf{P}^{w}[i, j]$ are the control points. In this paper, the superscript $w$ always denotes four-dimensional vectors in homogeneous space, and the corresponding notations without $w$ represent their three-dimensional Euclidean projections. A pair of square brackets denotes the index of the control points of NURBS. The B-spline basis functions $\tilde{N}_{i, p}(u)$ and $N_{j, q}(v)$ have the same recursive form with the standard NURBS in Ref. [PT97a]. The only difference is that in $u$-direction, or the so-called periodic direction, the knot sequence $\tilde{U}$ is infinite and periodic. We denote a complete nondecreasing subsequence of it in one period by $U=$ $\left\{u_{0}, u_{1}, \ldots, u_{m}\right\}$. In the other direction, the knot sequence $V=\left\{v_{0}, v_{1}, \ldots, v_{l}\right\}$ is a standard knot vector. Without loss of generality, we assume $u_{0}=0, v_{0}=v_{1}=\ldots=v_{q}=0$, $v_{l-q}=\ldots=v_{l-1}=v_{l}=1$ and the period of $\tilde{U}$ is 1 .

The topology of a non-degenerate periodic NURBS surface, as well as its control quads, is usually isomorphic with a cylindrical surface. It has two opposite periodic boundary edges as shown in Figure 2(a). We can construct a capshaped polar NURBS surface by shrinking a boundary edge of it to a single degenerate point in the center. The control mesh of it is in the polar configuration [MP11], which has triangular fans surrounding the the central pole. Formally, we denote the pole by $\mathbf{P}_{0}=\mathbf{P}[i, 0]$ for all valid $i$. As shown in Figure 2(b), a polar NURBS surface are isomorphic with a cap or a disk, and therefore, it can be used to represent some cap-shaped features, which are usually difficult to be represented by untrimmed standard NURBS surfaces with four boundary edges. Figure 1 shows an example.

Further analysis indicates that a $p$-degree $\mathrm{B}$-spline basis function is $C^{p-k}$ continuous at a knot with multiplicity $k$, which denotes the number of overlapping knots of the same value. However the polar NURBS surface has an exception at the degenerate pole: the polar curvature continuity also depends on the two rings of the surrounding control points besides the multiplicities of the knots. In order to focus on this

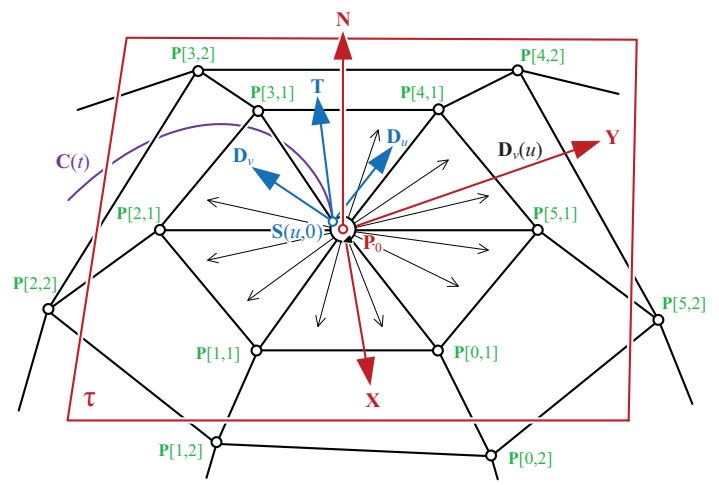

Figure 4: Polar continuity. $\mathbf{P}[i, j](i=0,1, \ldots, 5 ; j=1,2)$ are the control points. The surrounding black arrows show the radial cross-boundary derivatives. $\mathbf{X}$ and $\mathbf{Y}$ span the tangent plane $\tau$ of the pole $\mathbf{P}_{0} . \mathbf{T}$ is an arbitrary direction on the local plane spanned by $\mathbf{D}_{u}$ and $\mathbf{D}_{v}$ at parametric point $\left(u_{0}, 0\right)$, and the radial curve $\mathbf{C}(t)$ is in that direction.

special case and study the relation between polar curvature continuity and the zeroth-, the first- and the second-order derivatives determined by these control points, we assume that the multiplicities of the knots satisfy $C^{2}$ condition of the corresponding basis functions.

\section{Condition of curvature continuity at the pole}

First, we introduce a simplified notation of the derivatives.

$$
\mathbf{D}_{\underbrace{u \ldots u}_{r}}^{u} \underbrace{\nu \ldots \nu}_{s}(u)=\partial^{r+s} \mathbf{S}(l, v) /\left.\partial l^{r} \partial v^{s}\right|_{\substack{l=u \\ v=0}}
$$

We omit the parameter " $(u)$ " in the following derivation. A polar NURBS surface $\mathbf{S}(u, v)$ has a degenerate isoparametric curve $\mathbf{S}(u, 0)=\mathbf{P}_{0}$ for all valid $u$. At point $\mathbf{S}(u, 0)$, normal $\left(G^{1}\right)$ continuity requires that all the derivatives $\mathbf{T}=\alpha \mathbf{D}_{u}+$ $\beta \mathbf{D}_{v}(\forall \alpha, \beta \in \mathbb{R}$ ) should be on the polar tangent plane $\tau$ (see Figure 4). So there exists a unit normal vector $\mathbf{N}$ that satisfies $\mathbf{T} \cdot \mathbf{N}=0$. Since $\mathbf{P}[*, 0]$ (“*” denotes all valid indices) shrink to $\mathbf{P}_{0}$, we have $\mathbf{D}_{u} \equiv \mathbf{D}_{u u} \equiv \mathbf{0}$ according to the derivative formula on the boundary [PT97a]. It follows that $\mathbf{N} \cdot \mathbf{D}_{v}=0$. The NURBS derivative formula also presents that the radial derivative $\mathbf{D}_{v}$ is a linear combination of the pole and the first ring of the control points $\mathbf{P}[*, 1]$. So we have the following sufficient and necessary condition of $G^{1}$ polar continuity.

Condition 1 The polar NURBS surface is $G^{1}$ continuous at the pole if and only if the control points $\mathbf{P}[i, 0]$ and $\mathbf{P}[i, 1]$ are coplanar for all valid $i$.

Remark. Strictly, this paper only considers ordinary manifolds that have neither overlap, fold or normal flip. We can simply guarantee this by assuming that $\left(\left(\mathbf{P}[i, 1]-\mathbf{P}_{0}\right) \times\right.$ $\left.\left(\mathbf{P}[(i+1) \bmod n, 1]-\mathbf{P}_{0}\right)\right) \cdot \mathbf{N}>0$, where $n$ is the number of control points in the periodic direction. 
For curvature continuity, we first assume $\mathbf{P}[*, 1] \neq \mathbf{P}_{0}$ to avoid the degenerate case: $\mathbf{D}_{v}=\mathbf{0}$. The $G^{1}$ continuity condition guarantees the existence of two vectors $\mathbf{X}$ and $\mathbf{Y}$ satisfying $\mathbf{D}_{v}(u)=\alpha(u) \mathbf{X}+\beta(u) \mathbf{Y}$, where $\alpha(u)$ and $\beta(u)$ are scalar functions (see Figure 4). As a manifold without overlaps, any direction on the polar tangent plane has a unique corresponding parameter $u$ satisfying that equation. So on the parametric point $(u, 0)$ with the corresponding spatial direction $\mathbf{D}_{v}(u)$, the normal curvature $K$ of the curve $\mathbf{C}(t)=$ $\mathbf{S}(\gamma \cdot t+u, \delta \cdot t)$ can be formulated as follows according to the definition and the expanding rule of the derivative of composite functions.

$$
\begin{aligned}
K(u, \gamma, \delta) & =\left.\frac{\partial^{2} \mathbf{C}(t) / \partial t^{2} \cdot \mathbf{N}}{\partial \mathbf{C}(t) / \partial t \cdot \partial \mathbf{C}(t) / \partial t}\right|_{t=0} \\
& =\frac{\gamma^{2} \mathbf{D}_{u u} \cdot \mathbf{N}+2 \gamma \delta \mathbf{D}_{u v} \cdot \mathbf{N}+\delta^{2} \mathbf{D}_{v v} \cdot \mathbf{N}}{\gamma^{2} \mathbf{D}_{u} \cdot \mathbf{D}_{u}+2 \gamma \delta \mathbf{D}_{u} \cdot \mathbf{D}_{v}+\delta^{2} \mathbf{D}_{v} \cdot \mathbf{D}_{v}} .
\end{aligned}
$$

Note that we already have $\mathbf{D}_{u}=\mathbf{D}_{u u}=\mathbf{0}$. It follows that $\mathbf{D}_{u u} \cdot \mathbf{N}=0, \mathbf{D}_{u v} \cdot \mathbf{N}=0, \mathbf{D}_{u} \cdot \mathbf{D}_{u}=0$ and $\mathbf{D}_{u} \cdot \mathbf{D}_{v}=0$. So we have $K(u, \gamma, \delta)=\left(\mathbf{D}_{v v} \cdot \mathbf{N}\right) /\left(\mathbf{D}_{v} \cdot \mathbf{D}_{v}\right)$. The directional normal curvature $K$ is a constant at $\mathbf{S}(u, 0)$. No matter how to choose $\gamma$ and $\delta$, the polar curvature $K$ only depends on the parameter $u$, as well as the corresponding derivatives $\mathbf{D}_{v}(u)$ and $\mathbf{D}_{v v}(u)$. That is,

$$
K(u)=\left(\mathbf{D}_{v v}(u) \cdot \mathbf{N}\right) /\left(\mathbf{D}_{v}(u) \cdot \mathbf{D}_{v}(u)\right) .
$$

According to the definition of geometric continuity, a surface is $G^{k}$ continuous at a specified point if and only if there exists a reparameterization that can reform the surface to $C^{k}$ continuous locally. So the sufficient and necessary condition of curvature continuity at the pole is that there exists a $C^{2}$ continuous parametric surface $\hat{\mathbf{S}}(x, y)$ that osculates the pole in order two. We suppose that the local frame of the osculation surface is $\left(\mathbf{P}_{0}, \mathbf{X}, \mathbf{Y}\right)$. That is, $\partial \hat{\mathbf{S}} / \partial x=\mathbf{X}$ and $\partial \hat{\mathbf{S}} / \partial y=\mathbf{Y}$ at $\mathbf{P}_{0}$. Then, in the spatial direction $\mathbf{D}_{v}(u)=\alpha(u) \mathbf{X}+\beta(u) \mathbf{Y}$, the directional normal curvature is as follows.

$$
K(u)=\frac{\alpha^{2}(u) \mathbf{L} \cdot \mathbf{N}+2 \alpha(u) \beta(u) \mathbf{M} \cdot \mathbf{N}+\beta^{2}(u) \mathbf{R} \cdot \mathbf{N}}{\alpha^{2}(u) \mathbf{X} \cdot \mathbf{X}+2 \alpha(u) \beta(u) \mathbf{X} \cdot \mathbf{Y}+\beta^{2}(u) \mathbf{Y} \cdot \mathbf{Y}},
$$

where $\mathbf{L}=\partial^{2} \hat{\mathbf{S}} / \partial x^{2}, \mathbf{M}=\partial^{2} \hat{\mathbf{S}} / \partial x \partial y$ and $\mathbf{R}=\partial^{2} \hat{\mathbf{S}} / \partial y^{2}$ at $\mathbf{P}_{0}$. The osculation surface is $C^{2}$ continuous if and only if the three second-order partial derivatives $\mathbf{L}, \mathbf{M}$ and $\mathbf{R}$ are unique, respectively. Note that the denominator part equals $\mathbf{D}_{v}(u) \cdot \mathbf{D}_{v}(u)$, and therefore, combining (2) and the nondegenerate assumption of $\mathbf{D}_{v}(u)$, we have

$\alpha^{2}(u) \mathbf{L} \cdot \mathbf{N}+2 \alpha(u) \beta(u) \mathbf{M} \cdot \mathbf{N}+\beta^{2}(u) \mathbf{R} \cdot \mathbf{N}=\mathbf{D}_{v v}(u) \cdot \mathbf{N}$.

We introduce two scalar functions $\xi(u)$ and $\phi(u)$ to remove ".N" in each items. That is,

$$
\begin{aligned}
\mathbf{D}_{v v}(u)= & \alpha^{2}(u) \mathbf{L}+2 \alpha(u) \beta(u) \mathbf{M}+\beta^{2}(u) \mathbf{R}+ \\
& \xi(u) \mathbf{X}+\phi(u) \mathbf{Y},
\end{aligned}
$$

or the equivalent form with three scalars $L, M$ and $R$

$$
\mathbf{D}_{v v}(u)=\left(\alpha^{2}(u) L+2 \alpha(u) \beta(u) M+\beta^{2}(u) R\right) \cdot \mathbf{N}+
$$

$$
\xi(u) \mathbf{X}+\phi(u) \mathbf{Y} .
$$

So we have the following sufficient and necessary condition of curvature continuity at the pole.

Condition 2 The polar NURBS surface $\mathbf{S}(u, v)$ is curvature continuous at the pole if and only if

1. the surface satisfies the $G^{1}$ polar continuity condition;

2. with independent vectors $\mathbf{X}$ and $\mathbf{Y}$ (typically the two principle directions) satisfying $\mathbf{D}_{v}(u)=\alpha(u) \mathbf{X}+\beta(u) \mathbf{Y}$, there exist three vectors satisfying (4), or three scalars satisfying (5).

\section{Construction of a polar NURBS surface with curvature continuity}

A $(p, q)$-degree polar NURBS surface is $G^{2}$ continuous if it is a) $C^{2}$ continuous and b) $G^{2}$ continuous at the pole. For the first condition, we only need to ensure that the multiplicity of each knot of $U$ is not greater than $p-2$ and of $V$, not greater than $q-2(p, q \geq 3)$. Besides, as what geometric modeling systems usually do, we should also ensure that the control-point mesh has no superposition or flip. Then, the key problem is to construct the control points to satisfy the condition of polar curvature continuity.

According to the derivative properties of NURBS on the boundary, once $\mathbf{P}^{w}[*, 0]$ are fixed, the radial derivative curve $\mathbf{D}_{v}(u)$ and the first ring of control points $\mathbf{P}^{w}[*, 1]$ are interconvertible exactly (see Ref. [PT97a] §4.5, §5.3 and §5.5). For the same reason, $\mathbf{D}_{v v}(u)$ and $\mathbf{P}^{w}[*, 2]$ are interconvertible once $\mathbf{P}^{w}[*, 0]$ and $\mathbf{P}^{w}[*, 1]$ are fixed. So instead of the control points, we discuss the first- and the second-order radial derivatives $\mathbf{D}_{v}(u)$ and $\mathbf{D}_{v v}(u)$ as symbols, since they are equivalent to the corresponding control points. This symbolic method helps to unify the formulae of NURBS and NUPBS.

In practice, there are two cases when the pole is prescribed: the first ring of the control points (or $\mathbf{D}_{v}(u)$ ) are specified, or not. For the case that $\mathbf{D}_{v}(u)$ is not given, we first discuss how to construct $\mathbf{D}_{v}(u)$ to satisfy $G^{1}$ continuity. We freely specify $\mathbf{N}$, the unit normal vector of the pole. Then, we choose two linearly independent vectors $\mathbf{X}$ and $\mathbf{Y}$ to satisfy $\mathbf{X} \cdot \mathbf{N}=0$ and $\mathbf{Y} \cdot \mathbf{N}=0$, which span the tangent plane $\tau$ at the pole (see Figure 4). Condition 1 implies that

$$
\mathbf{D}_{v}(u)=\alpha(u) \mathbf{X}+\beta(u) \mathbf{Y},
$$

where $\alpha(u)$ and $\beta(u)$ are scalar periodic NURBS functions, and the constant vectors $\mathbf{X}$ and $\mathbf{Y}$ also can be treated as onedegree one-control-point periodic NURBS curves. With the symbolic operators of addition and scalar multiplication of NURBS [PT97b], we directly compute the NURBS form of $\mathbf{D}_{v}(u)$. Its degree is the maximum of the degrees of $\alpha(u)$ and $\beta(u)$. The introduction of the symbolic operators here makes the formulae simple and clear, and it prevents the readers from being lost in the detailed implementation. 
$\alpha(u)$ and $\beta(u)$ can be arbitrary. An ellipse-shaped radial derivative curve like the one in Figure 4 can be formulated by $\mathbf{D}_{v}(u)=\cos (u) \mathbf{X}+\sin (u) \mathbf{Y}$. Compared with (6), we choose $\alpha(u) \approx \cos (u)$ and $\beta(u) \approx \sin (u)$. The quality of approximation does not interfere with continuity however only has slight consequences for the shape. So for simplicity, we usually use a uniform knot vector and the same weight of control points to construct $\alpha(u)$ and $\beta(u)$. For example, the control points of $\alpha(u)$ and $\beta(u)$ can be $\{0,1,0,-1\}$ and $\{1,0,-1,0\}$, respectively. More strictly, the trigonometric functions can be exactly represented by NURBS after the following reparameterization: $\sin (u)=2 t /\left(1+t^{2}\right)$, $\cos (u)=\left(1-t^{2}\right) /\left(1+t^{2}\right)$ where $t=\tan (u / 2)$ (see Ref. [PT97a] §7.5). Of course, $\mathbf{D}_{v}(u)$ also can be designed manually satisfying Condition 1 . Backwardly, $\alpha(u)$ and $\beta(u)$ are retrieved from $\mathbf{D}_{v}(u)$ (or indirectly from the first ring of control points). Once $\mathbf{D}_{v}(u), \mathbf{X}$ and $\mathbf{Y}$ are determined, we have $\alpha[i] \mathbf{X}+\beta[i] \mathbf{Y}=\mathbf{D}_{v}[i]$ for each control point with index $i$. It follows that $[\alpha[i], \beta[i], 0]^{\mathrm{t}}=[\mathbf{X Y N}]^{-1} \mathbf{D}_{v}[i]$. Both $\alpha(u)$ and $\beta(u)$ share the same degree, weights and knots with $\mathbf{D}_{v}(u)$.

Then we discuss the construction of $\mathbf{D}_{v v}(u)$ assuming that $\mathbf{D}_{v}(u)$ (or $\mathbf{P}^{w}[*, 1]$ ) has been determined. Condition 2 gives the construction formulae (4) and (5), where $\xi(u)$ and $\phi(u)$ are arbitrary scalar periodic NURBS curves. They involve the symbolic operations of addition, inner product and scalar multiplicity of NURBS [PT97b], and the final result of $\mathbf{D}_{v v}(u)$ still preserves the NURBS form. Its degree is the maximum of the twice of the degree of $\mathbf{D}_{v}(u)$, the degree of $\xi(u)$ and the degree of $\phi(u)$. Note if $\{\mathbf{X}, \mathbf{Y}, \mathbf{N}\}$ form an orthonormal basis, (3) can be reduced to the following equation by assuming that $M=0$.

$$
K(u)=\frac{\alpha^{2}(u) L+\beta^{2}(u) R}{\alpha^{2}(u)+\beta^{2}(u)} .
$$

At a prescribed parametric point $u$, the curvature $K(u)$ is a positive combination of $L$ and $R$. In non-degenerate cases, the continuous curves $\alpha(u)$ and $\beta(u)$ have different zero points. So the assumption of $M$ ensures that $L$ and $R$ are the maximum and the minimum of the curvature function $K(u)$, which are the so-called principle curvatures, respectively. Thus, we can use that formula to construct $\mathbf{D}_{v v}(u)$ by principle curvatures. For $\xi(u)$ and $\phi(u)$, we usually first ignore them in the construction of $\mathbf{D}_{v v}(u)$, and then move the control points of $\mathbf{D}_{v v}(u)$ along the tangent plane spanned by $\mathbf{X}$ and $\mathbf{Y}$. This method assumes that $\Delta(u)=\xi(u) \mathbf{X}+\phi(u) \mathbf{Y}$ shares the same degree, weights and knot vector with $\mathbf{D}_{v v}(u)$. So the addition of $\Delta(u)$ to $\mathbf{D}_{v v}(u)$ can be reduced to pointwise additions of the control points.

Once $\mathbf{P}_{0}, \mathbf{D}_{v}(u)$ and $\mathbf{D}_{v v}(u)$ are determined, the three central rings of control points can be constructed according to the derivative formulae of NURBS surface on the boundary [PT97a]. In practice, the radial derivatives are usually polynomial (for example, $n$-sided hole filling), and in these cases, the conversion algorithm from radial derivative curves to control points can be as simple as follows.

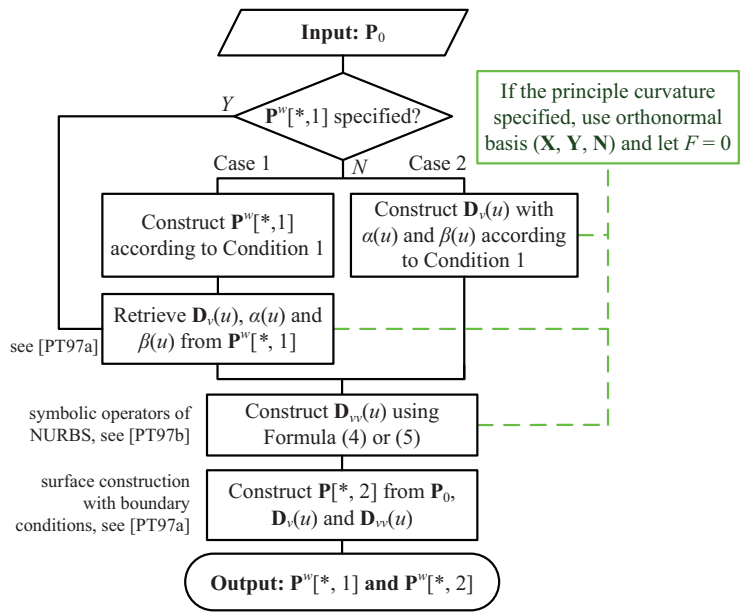

Figure 5: The flow chart of the construction method.

- Form a one-degree periodic NURBS curve $\mathbf{D}_{o}(u)$ with only one control point $\mathbf{P}_{0}$.

- Elevate the degrees of the curves $\mathbf{D}_{o}(u), \mathbf{D}_{v}(u)$ and $\mathbf{D}_{v v}(u)$ to the maximum degree of them, denoted by $p$.

- Compute the union of the knot vectors of the three radial derivative curves, denoted by $\bar{U}$, and then ensure they share the same knot vector $\bar{U}$ by knot refinement (so the numbers of control points are also the same).

- For the $k$ th ring of the control points of the surface, we use the same weight $w_{k}$. For each column $i$, the three control points $\mathbf{P}[i, 0], \mathbf{P}[i, 1]$ and $\mathbf{P}[i, 2]$, as well as the corresponding weights $w_{0}, w_{1}$ and $w_{2}$, form the starting part of a NURBS curve with degree $q$ and knot vector $V$ that come from the radial direction of the polar NURBS surface. Then, the three control points can be solved by Hermite interpolation using $\mathbf{D}_{o}[i], \mathbf{D}_{v}[i]$ and $\mathbf{D}_{v v}[i]$ as boundary conditions at $v=0$.

The entire construction process is summarized in Figure 5. Our method employs the Hermite interpolation algorithm of NURBS surface, which aims to compute the control points of the result surface column by column, with the control points of the specified cross-boundary derivatives. If $\mathbf{D}_{v}(u)$ and $\mathbf{D}_{v v}(u)$ are rational, the formulae in the last step will be more complicated in implementation. We do not discuss the details of it here since it is a fundamental operation of construction of NURBS surface [PT97a].

\section{Reforming an ordinary polar NURBS surface to curvature continuous}

The reformation algorithm aims to achieve curvature continuity at the pole by moving the control points locally. First we assume that the pole $\mathbf{P}_{0}$ and the first ring of the control points are coplanar so that $G^{1}$ continuity is guaranteed according to Condition 1 . If not, we only need to project 
the control points onto an estimated polar tangent plane. So the local frame $\{\mathbf{X}, \mathbf{Y}, \mathbf{N}\}$ at the pole can be constructed by choosing two independent vectors $\mathbf{X}$ and $\mathbf{Y}$ that are perpendicular to the polar unit normal vector $\mathbf{N}$. Then, $\mathbf{D}_{v}(u)$ and $\mathbf{D}_{v v}(u)$, as well as $\alpha(u)$ and $\beta(u)$, can be retrieved from the three central rings of the control points. We decompose $\mathbf{D}_{v v}(u)$ into two orthogonal parts: the scalar normal part $D_{v v}(u)$ and the tangential part $\Delta(u)$. That is,

$$
\mathbf{D}_{v v}(u)=D_{v v}(u) \cdot \mathbf{N}+\Delta(u), \Delta(u) \cdot \mathbf{N} \equiv 0 .
$$

The NURBS curves $D_{v v}(u)$ and $\Delta(u)$ share the same degree, weights and knot vector with $\mathbf{D}_{v v}(u)$, and pointwise, $D_{v v}[i]=\mathbf{D}_{v v}[i] \cdot \mathbf{N}$ and $\Delta[i]=\mathbf{D}_{v v}[i]-D_{v v}[i] \cdot \mathbf{N}$ for all valid $i$. Compared with (5), the surface is curvature continuous if and only if there exist three scalar functions satisfying

$$
D_{v v}(u) \equiv \alpha^{2}(u) L+2 \alpha(u) \beta(u) M+\beta^{2}(u) R .
$$

(8) is an alternative form of (5) in Condition 2. A randomly constructed polar NURBS surface usually fails to achieve curvature continuity because this equation can be rarely satisfied without careful construction of the second-order radial derivative curve $D_{v v}(u)$.

The satisfaction of (8) depends on all valid $u$. Fortunately, the NURBS form of the scalar functions allows this problem to be converted to finite times of verification of the following equation about the control points.

$$
D_{v v}[i]=\left(\alpha^{2}\right)[i] \cdot L+2(\alpha \beta)[i] \cdot M+\left(\beta^{2}\right)[i] \cdot R, \forall i,
$$

where the NURBS functions $D_{v v},\left(\alpha^{2}\right),(\alpha \beta)$ and $\left(\beta^{2}\right)$ share the same degree, weights and knot vector after degree elevation, knot refinement and uniformization of denominators. $\mathbf{D}_{v}(u)$ of an ordinary surface has at least three independent control points (or, the surface will be ill conditioned). So the number of rows of the linear system (9) is no less than the number of the unknowns ( $L, M$ and $R$ ). Thus, the surface is curvature continuous at the pole if and only if the linear system has a unique solution (where all equations are satisfied).

Otherwise, the overdetermined system only has a leastsquare solution $\{\bar{L}, \bar{M}, \bar{R}\}$ by Moore-Penrose pseudoinverse [Moo20], and that gives an approximation to the discontinuous pole. Substitute them to (8) and then to (7), we can construct $\mathbf{D}_{v v}(u)$ to satisfy polar curvature continuity. The final control points can be computed as the previous section. A shortcoming of this method is the introduction of degree elevation and knot insertion, which may cause the increment of the number of control points. However this is necessary if we need to exactly preserve the control points of the first ring, as well as the pole. Otherwise, we should simplify $\alpha(u)$ and $\beta(u)$ before the computation of (8). Furthermore, if we want to preserve the number of the control points of the original surface, as well as their weights and knot vectors, it is also practical to slightly move the control points of the second ring to fit $\mathbf{D}_{v v}(u)$ to the ideal $\mathbf{D}_{v v}(u)$ that satisfies polar curvature continuity. Both these fitting and simplification
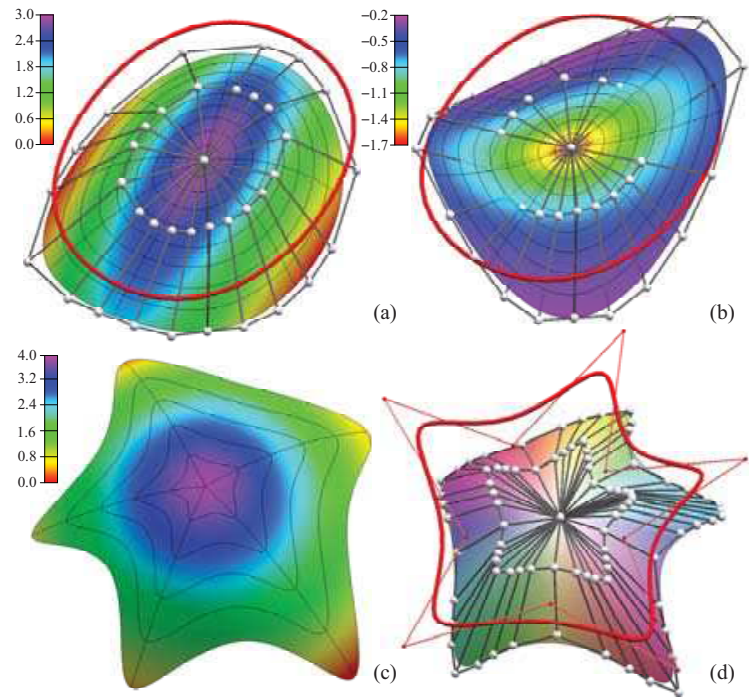

Figure 6: Curvature continuous polar NURBS surfaces. They have (a) ellipsoidal, (b) saddle and $(c)(d)$ spherical poles, respectively. The red curves are the first order radial directive curves $\mathbf{D}_{v}(u)$. The Gaussian curvature charts in (a), (b) and (c) show their continuity.

methods may introduce an error. The choice of strategy depends on the application and the balance between precision and simplicity. We do not expand the very details here since they are surely beyond the limit of this paper.

\section{Examples and applications}

We first present examples of the results by our method. The constructive method proposed in Section 5 is used to generate free-shape surfaces. Figure 6 demonstrates three cases, which have an ellipsoidal (two different principle curvatures with the same sign), a saddle (principle curvatures with different signs) and a spherical (same principle curvatures) pole, respectively. The curvature charts illustrate the smoothness and continuity of the shape. In order to ensure $C^{2}$ continuity in the periodic direction, the first-order radial derivative curves are cubic. So the degrees of the final surfaces are all $(6,3)$, which are acceptable in CG and CAD.

In Figure 7, we reform a non- $G^{2}$ polar NURBS surface to $G^{2}$ continuous. The algorithm uses the pole and the first ring of the control points to derive the zeroth- and the first-order radial derivative curves. The second-order radial derivative curve is then constructed according to (7) and (8). After ensuring the consistence of the degree and the knots of it with the periodic direction of the surface, the new positions of the control points of the second ring are then solved. In this case, the degree of the first-order radial derivative curve can be reduced to 3 . Formula (8) doubles it to 6 , so the final surface has degree 6 in the periodic direction. 


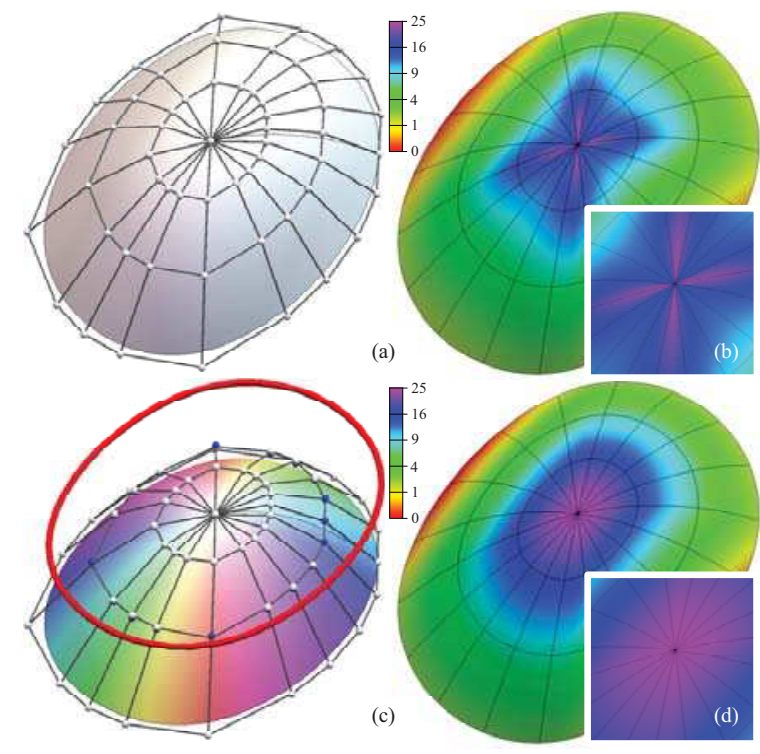

Figure 7: Reforming a non- $G^{2}$ polar NURBS surface to $G^{2}$. (a) shows the control points of the original discontinuous surface, while (b) presents its Gaussian curvature chart. Note that around the pole, the Gaussian curvatures converge to different values in different directions. (c) shows the control points of the reformed surface. The blue dots are the original control points before moving, and the red curve represents the first-order radial derivative. The Gaussian curvature chart in $(d)$ illustrates its $G^{2}$ continuity.

Our $G^{2}$ cap-like surface is also proved to be a powerful tool in filling holes, which aims to provide one or more surface patches to interpolate the hole's boundary and crossboundary derivatives with continuity constraints. For example, a recent work [SYSP11] used a polar NURBS surface to cap a hole that has a loop-shaped boundary. This method avoids the trimmed domain and is more CADfriendly than the other methods based on blending or subdivision [GLZ90, NSY09, Rei98, SYSP10]. However it also suffers from the problem of polar curvature continuity. As a practical application, the following example demonstrates how our method solves this continuity problem. In Figure 8, we combine the methods in [SYSP11] and in this paper to generate a $G^{2}$ polar NURBS surface that contacts the specified five boundary surfaces with curvature continuity. We achieves the curvature continuous pole with non-zero Gaussian curvature compared to the artificial flat pole by their method. Figure 9 shows a mechanical model in which we fill the hole of the rounded tip of each propeller blade with a single $G^{2}$ polar NURBS surface. The boundary of the hole, which is reconstructed from sectional views, is a quartic NURBS curve that has non-uniform knots to represent the sharp feature of the blade.
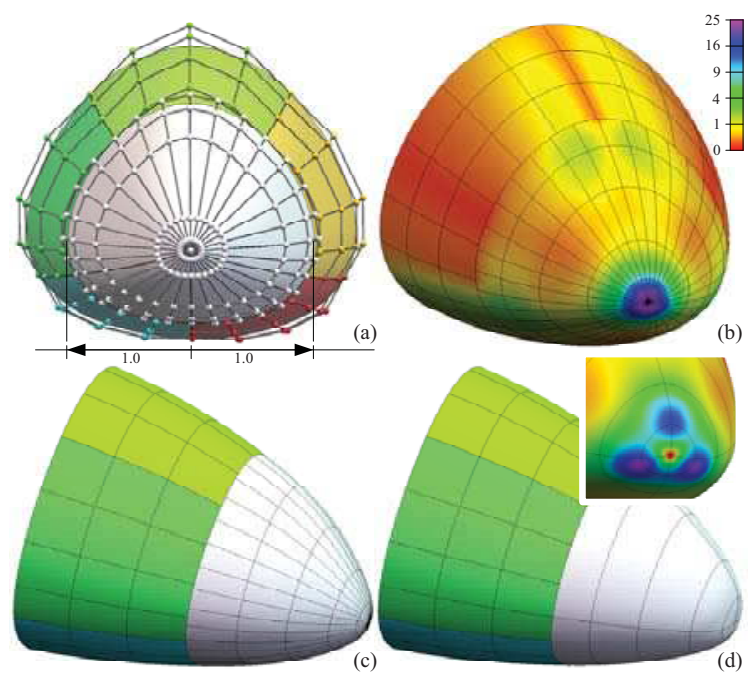

Figure 8: Capping a loop-shaped five-sided hole with a $G^{2}$ continuous periodic NURBS surface. (a) shows the control points of the boundary surfaces (chromatic) and the filling surface (white), respectively; the Gaussian curvature chart in (b) presents $G^{2}$ continuity; the following comparison is between our algorithm (c) and Ref. [SYSP11] (d).
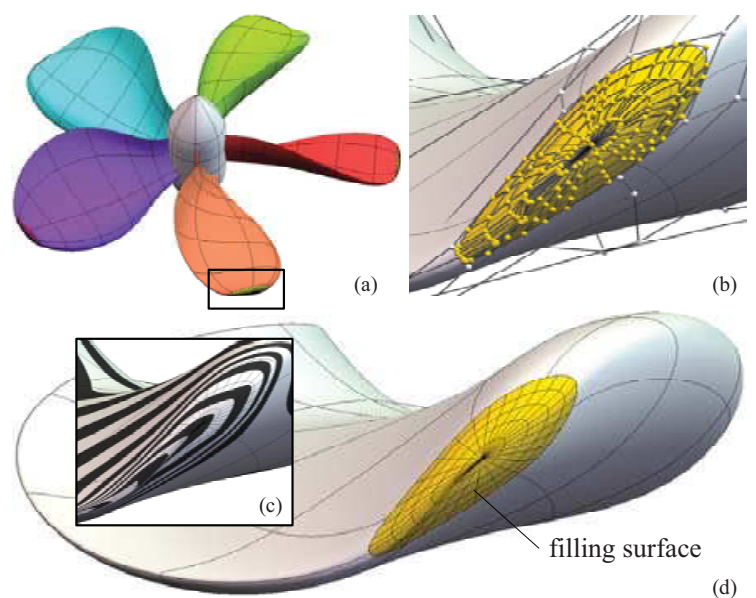

(d)

Figure 9: The tip of each blade of the propeller in (a) is filled with a single $G^{2}$ polar NURBS surface (see the continuous reflection stripes in (c)) which has non-uniform knots in the periodic direction (denser near the sharp part), $(40 \times 6)$ control points $(c)$ and degrees $(8,5)$.

In particular, the zeroth-, the first- and the second-order periodic cross-boundary derivatives $\mathbf{B}_{h}(u)(h=o, v, v v)$ are first constructed by joining the cross-boundary derivatives from each boundary surfaces after reparameterization [SYSP11]. Then we estimate the central point $\mathbf{O}$ and the central normal vector $\mathbf{N}$ by the original algorithm. As sim- 
ply as we can, we form the first-order radial derivative curve $\mathbf{D}_{v}(u)$ in periodic NURBS using the same degree and knot vector with the boundary $\mathbf{B}_{o}(u)$. After that, we estimate the control points of $\mathbf{D}_{v}(u)$ to achieve a better shape. For each control point of $\mathbf{D}_{v}(u)$, we project the corresponding control point of $\mathbf{B}_{o}(u)$ onto the polar tangent plane, and let it be the vector from $\mathbf{O}$ to the projection point. That is,

$$
\mathbf{D}_{v}[i]=\left(\mathbf{B}_{o}[i]-\mathbf{O}\right)-\left(\left(\mathbf{B}_{o}[i]-\mathbf{O}\right) \cdot \mathbf{N}\right) \cdot \mathbf{N},
$$

for all valid $i$. After choosing an arbitrary orthonormal basis $\{\mathbf{X}, \mathbf{Y}, \mathbf{N}\}$, we can retrieve the scalar functions $\alpha(u)$ and $\beta(u)$, which have the same degree, weights and knot vector with $\mathbf{D}_{v}(u)$. The three parameters $L, M$ and $R$ can be specified or estimated by finding a least-square fitting to the normal curvatures of each parabola through $\mathbf{B}_{o}[i], \mathbf{B}_{o}^{\tau}[i]$ and $\mathbf{O}$. That is, $K[i]=2\left(\left(\mathbf{B}_{o}[i]-\mathbf{O}\right) \cdot \mathbf{N}\right) /\left(\mathbf{D}_{v}[i] \cdot \mathbf{D}_{v}[i]\right)$. According to the formula of directional curvature (3), we simply have the following linear system of $L, M$ and $R$.

$$
K[i](\alpha[i]+\beta[i])^{2}=\alpha[i]^{2} \cdot L+2 \alpha[i] \beta[i] \cdot M+\beta[i]^{2} \cdot R,
$$

for all valid $i$. Use Moore-Penrose pseudoinverse to get the least-square solution $\{\bar{L}, \bar{M}, \bar{R}\}$ and then construct $\mathbf{D}_{v v}(u)$ as the previous section. After degree elevation and knot refinement to ensure the consistence of the curves of boundary conditions $\mathbf{D}_{h}(u)$ and $\mathbf{B}_{h}(u)(h=o, v, v v)$, we can generate the final surface by the method in Ref. [SYSP11].

The algorithm in Ref. [SYSP11] assumes the zero polar curvature to guarantee polar curvature continuity. It leads to a flat region at the pole and a high-curvature area around the pole (see Figure 8(d)). The comparison of the distribution of Gaussian curvature illustrates that the non-flat pole improves the quality (smoothness) of the surface. Note the control meshes of the boundary surfaces may not coincide with the filling surface although they share the same boundary geometrically. This is common in NURBS surface modeling for two possible reasons: 1) different degrees of NURBS, and 2) different parameterization. The final surface is friendlier to CAD since periodic NURBS surfaces are interconvertible with standard NURBS exactly (the control points in all figures are of the corresponding standard NURBS).

\section{Conclusion}

We proposes a sufficient and necessary condition of curvature continuity at the pole of a polar NURBS surface. Based on that criterion, we present two constructive algorithms to generate a polar NURBS surface with curvature continuity and to reform a polar NURBS surface to be curvature continuous. These methods focus on more general cases compared to [MP09, MP11] since for our methods, there are no limitations on the degree, the knot vector, as well as the weights, of the input data. Formulation in this paper only involves the symbolic representation and operations of NURBS rather than control points or subdivision formulae. Besides, a polar NURBS surface can be directly and precisely converted to standard NURBS without elevating the degree or introducing a tolerance. The methods are compatible with existing CAD/CAM systems.

\section{References}

[Far97] FARIN G.: Curves and surfaces for computer-aided geometric design: a pratical guide, 4th ed. Elsevier Science \& Technology Books, 1997. 1

[GLZ90] Gregory J., LAU V., Zhou J.: Smooth parametric surfaces and $n$-sided patches. In Computation of Curves and Surfaces, Dahmen W., Gasca M., Micchelli C., (Eds.), vol. 307 of NATO ASI Series. Springer Netherlands, 1990, pp. 457-498. 7

[KMP06] Karčiauskas K., Myles A., Peters J.: A $C^{2}$ polar jet subdivision. In Proceedings of the fourth Eurographics symposium on Geometry processing (Aire-la-Ville, Switzerland, Switzerland, 2006), SGP '06, Eurographics Association, pp. 173-180. 2

[KP07] KarČIAUSKas K., Peters J.: Bicubic polar subdivision. ACM Transactions on Graphics 26, 4 (Oct. 2007), 14:114:8. 2

[KP09] KarČIaUskas K., Peters J.: Guided spline surfaces. Computer Aided Geometric Design 26, 1 (2009), 105-116. 2

[KR90] K. REED D. HARROD W. C.: Initial graphics exchange specification (IGES) version 5.0. U.S. Dept. of Commerce, National Bureau of Standards, 1990. 1

[Lev06] LEVIN A.: Modified subdivision surfaces with continuous curvature. ACM Transactions on Graphics 25, 3 (July 2006), 1035-1040. 2

[Moo20] Moore E. H.: On the reciprocal of the general algebraic matrix. Bulletin of the American Mathematical Society 26, 9 (1920), 394-395. 6

[MP09] Myles A., Peters J.: Bi-3 $C^{2}$ polar subdivision. ACM Transactions on Graphics 28, 3 (July 2009), 48:1-48:12. 2, 8

[MP11] Myles A., Peters J.: $C^{2}$ splines covering polar configurations. Computer-Aided Design 43, 11 (2011), 1322-1329. $1,2,3,8$

[NSY09] NASRI A., SABIN M., YASSEEN Z.: Filling $n$-sided regions by quad meshes for subdivision surfaces. Computer Graphics Forum 28, 6 (2009), 1644-1658. 7

[Pra97] Prautzsch H.: Freeform splines. Computer Aided Geometric Design 14, 3 (1997), 201-206. 2

[PT97a] PIEgl L. A., TILler W.: The NURBS book, 2nd ed. Springer, 1997. 1, 2, 3, 4, 5

[PT97b] PIEGL L. A., TIller W.: Symbolic operators for NURBS. Computer-Aided Design 29, 5 (1997), 361-368. 2, 4,5

[Rei98] REIF U.: TURBS - topologically unrestricted rational Bsplines. Constructive Approximation 14, 1 (1998), 57-77. 2, 7

[SYSP10] SHI K.-L., Yong J.-H., SUN J.-G., PAUl J.-C.: $G^{n}$ blending multiple surfaces in polar coordinates. Computer-Aided Design 42, 6 (2010), 479-494. 2, 7

[SYSP11] ShI K.-L., Yong J.-H., SUN J.-G., PAUL J.-C.: $G^{n}$ filling orbicular $N$-sided holes using periodic B-spline surfaces. Science China F 54, 7 (2011), 1383-1394. 1, 2, 7, 8 